\title{
HIV-1 Subtype distribution in morocco based on national sentinel surveillance data 2004-2005
}

\author{
Mohammed Akrim', Sanae Lemrabet ${ }^{1}$, Elmir Elharti ${ }^{2}$, Rebecca R Gray ${ }^{3}$, Jean Claude Tardy ${ }^{4}$, Robert L Cook ${ }^{5^{*}}$, \\ Marco Salemi ${ }^{6}$, Patrice Andre ${ }^{4}$, Taj Azarian ${ }^{5}$ and Rajae El Aouad ${ }^{1,2}$
}

\begin{abstract}
Background: Little is known about HIV-1 subtype distribution in Morocco. Some data suggest an emergence of new HIV subtypes. We conducted phylogenetic analysis on a nationally representative sample of $60 \mathrm{HIV}-1$ viral specimens collected during 2004-2005 through the Morocco national HIV sentinel surveillance survey.

Results: While subtype $B$ is still the most prevalent, $23.3 \%$ of samples represented non-B subtypes, the majority of which were classified as CRF02_AG (15\%). Molecular clock analysis confirmed that the initial introduction of HIV-1B in Morocco probably came from Europe in the early 1980s. In contrast, the CRF02_AG strain appeared to be introduced from sub-Saharan Africa in two separate events in the 1990s.

Conclusions: Subtype CRF02_AG has been emerging in Morocco since the 1990s. More information about the factors introducing HIV subtype-specific transmission will inform the prevention strategy in the region.
\end{abstract}

Keywords: HIV-1, subtypes, phylogeny, Morocco

\section{Introduction}

HIV-1 variability remains a formidable challenge for designing a protective vaccine or an effective cure. The HIV-1 is divided into 4 groups: M, N, O and P. Group $\mathrm{M}$ is responsible for the current pandemic and includes more than 49 circulating recombinant forms (CRFs), 9 subtypes, 5 sub-subtypes, and unique recombinant forms (URFs) [1,2]. HIV genetic diversity is generated by the high rate of virus mutation, rapid viral turnover and frequent recombination events between subtypes [3]. Furthermore, there is an unequal geographic distribution of HIV-1 subtypes and CRFs around the world characterized by different epidemic behaviours and growth rates [4]. For instance, in western Europe and North America, subtype B is the most prevalent whereas in sub-Saharan Africa subtypes A, C, D and CRF02_AG predominate [5-7]. This geographic distribution of HIV1 subtypes could result from migration, travel, or geographic accessibility. These factors may contribute to the transmission of these clades outside the regions

\footnotetext{
* Correspondence: cookrl@ufl.edu

${ }^{5}$ Department of Epidemiology and Emerging Pathogens Institute, University of Florida, Gainesville, USA

Full list of author information is available at the end of the article
}

where they are most prevalent $[8,9]$. The increasing diversity of HIV-1 underscores the need for diagnostics, patient monitoring tools, and treatment options that are effective across the full spectrum of known groups, subtypes, and recombinant forms.

The first reported case of HIV/AIDS in Morocco occurred in 1986. Up to December 2010, a cumulative total of 2,914 persons have been diagnosed with AIDS in Morocco, and estimates suggest approximately 26,000 persons are living with HIV in the country [10]. Among them, 58\% were identified during the 6 last years. Furthermore, more than half of cases are from 3 regions: the Agadir region (22\%), the Marrakech region $(16 \%)$ and the Casablanca region (14\%). Young adults (15-39 years) represent $64 \%$ of all the cases, and the proportion of HIV infections in women has increased from $18 \%$ (1986-1990) to more than 40\% (2004-2008). HIV-1 transmission is reportedly attributed to heterosexual transmission in more than $80 \%$ of individuals.

A national HIV sentinel surveillance network has been implemented in Morocco since 1993 [11]. This surveillance is based on an anonymous, unlinked study and is approved by the WHO Ethical Committee. Studied groups include pregnant women, patients consulting
C Biomed Central

C 2012 Akrim et al; licensee BioMed Central Ltd. This is an Open Access article distributed under the terms of the Creative Commons Attribution License (http://creativecommons.org/licenses/by/2.0), which permits unrestricted use, distribution, and reproduction in any medium, provided the original work is properly cited. 
healthcare centres with STIs, persons with tuberculosis, prisoners, injecting drug users (IDUs) and sex workers.

A study of HIV subtypes in Morocco from 1997 showed a predominance of HIV-1 subtype B (93.5\%), a pattern more similar to Europe than sub-Saharan Africa [12]. More recently, an analysis of HIV subtypes from a single region of Morocco suggests an increase in persons with HIV CRF02-AG, which has typically been associated with infections from sub-Saharan Africa [13]. Since the mid-1990s, Morocco has been experiencing a significant immigration of persons from sub-Saharan Africa, many of which are attempting to enter Europe. More recently, Spain and the European Union have intensified their border and coastal surveillance. Consequently, Morocco has shifted from being a transit country to the final station for many migrants. Other countries in the MENA region are also demonstrating a more diverse HIV epidemic in terms of HIV subtype distribution [14].

The study of HIV subtype distribution may reveal epidemiological patterns of transmission or distinct networks associated with specific risk behaviours. Phylogenetic analysis can further expand the identification of specific epidemiological clusters of HIV infection from a common origin [9]. We investigated the pattern of HIV-1 subtype diversity and high-resolution phylogenetic analysis of a representative sample of $60 \mathrm{HIV}-$ infected persons identified through the Morocco national HIV sentinel surveillance program.

\section{Materials and methods Sample collection}

Samples were collected as part of the sentinel surveillance system, a national HIV epidemic AIDS surveillance survey carried out each spring by the Moroccan Ministry of Health to assess trends in the HIV epidemic. The sera were collected from different regions of Morocco during 2004 and 2005. All of the $60 \mathrm{HIV}$-positive samples during the 2004-2005 survey were included in this study. Samples were screened HIV-1 positive by Elisa (HIV1/2 Genscreen plus, Bio-Rad, France) and confirmed by Western blot test (Genlabs, USA). These samples were representative of the different regions of Morocco and were consistent with the age and sex distribution of reported HIV cases within the country (Table 1).

\section{PCR and DNA sequencing}

To study diversity, samples were sequenced in pol gene region [protease gene $(P R)$ and $2 / 35$ ' region of the reverse transcriptase gene $(\mathrm{RT})]$. The viral RNA was extracted and PR and RT genes were RT-PCR amplified as previously described [15]. The fragments obtained were sequenced on both strands using an automated sequencer Beckman CEQ 2000 DNA Analysis System, and subtyped by using the Rega HIV-1 Subtyping tool version $2.0 \quad$ (http://dbpartners.stanford.edu/ RegaSubtyping).

GenBank accession numbers for the sequences reported in this study are JQ316543 to JQ316600 and JQ344156 to JQ344204 for PR and RT sequences respectively.

\section{Dataset assembling}

All sequences from Morocco were divided into protease $(\mathrm{PR}, \mathrm{N}=58)$ and reverse transcriptase $(\mathrm{RT}, \mathrm{N}=49)$ alignments (Table 1). A set of full genome reference sequences (Genbank:AF004885, Genbank:AB253421, Genbank:AB253429, Genbank:AF286241, Genbank: AF286237, Genbank:K03455, Genbank:AY423387, Genbank:AY173951, Genbank:AY331295, Genbank: DQ853463, Genbank:U52953, Genbank:U46016, Genbank:AF067155, Genbank:AY772699, Genbank:K03454, Genbank:AY371157, Genbank:AY253311, Genbank: U88824, Genbank:AF077336, Genbank:AF005494, Genbank:AF075703, Genbank:AJ249238, Genbank: AY371158, Genbank:AJ249236, Genbank:AJ249237, Genbank:AF377956, Genbank:AF084936, Genbank: AF061641, Genbank:U88826, Genbank:AF190127, Genbank:AF190128, Genbank:AF005496, Genbank: EF614151, Genbank:AF082394, Genbank:AF082395, Genbank:AJ249235, Genbank:AJ249239, Genbank: AY271690) were downloaded from the Los Alamos HIV database. An additional set of sequences of PR/RT for subtypes HIV-1 CRF02-AG and subtype B were downloaded from the Los Alamos database. The criterion for inclusion in this dataset included the following: sequences were published, were not amplified in culture and had a known location and year of sampling. The HIV-1B dataset was reduced for computational practicality and included representative sequences only from major geographic areas. Multiple-sequence alignments were obtained by codon-alignment with the CLUSTAL algorithm, and subsequently manually edited for optimization (Alignments are available from the authors upon request).

\section{Phylogenetic analysis}

Maximum likelihood (ML) trees were first inferred using the Moroccan sequences and full genome references sequences. Analyses were performed assuming the GTR + Gamma model of nucleotide evolution. Statistical support was assessed by non-parametric bootstrapping (number of replicates $=500$ ) using PHYML version 3.0 [16]. Sequences that clustered with a pure subtype with a bootstrap value of $>80$ were classified as such. Sequences that clustered with the CRF02_AG with a bootstrap value $>50$ were classified as HIV-1 
Table 1 Database of HIV sequences included in the study

\begin{tabular}{|c|c|c|c|c|c|c|c|}
\hline Sample Code & $\mathrm{N}^{\circ}$ & Origin & Risk Group & Sex & Age & Sub-type PR & Subtype RT \\
\hline N1BBB & $B$ & Tetouan & Patient with TB & M & 35 & $B$ & B \\
\hline$\overline{\mathrm{N} 1 \mathrm{CBB}}$ & $C$ & Tetouan & Patients with STI's & $M$ & 32 & $B$ & $B$ \\
\hline $\mathrm{N1AB}$ & $A$ & Tetouan & Patient with TB & $M$ & 39 & & $B$ \\
\hline N2NBB & $\mathrm{N}$ & Rabat & Male prisoners & M & 30 & $B$ & B \\
\hline $\mathrm{N} 238 \mathrm{~B}$ & 38 & Rabat & Female SW in prison & $F$ & 19 & $B$ & \\
\hline S4DBB & D & Casablanca & Patients with STI's & $F$ & 41 & $B$ & $B$ \\
\hline S4EBB & $E$ & Casablanca & Patient with TB & M & 39 & $B$ & $B$ \\
\hline S4Q1BB & Q1 & Casablanca & Pregnant women & $\mathrm{F}$ & 19 & $B$ & $B$ \\
\hline $\mathrm{S} 3 \mathrm{MBB}$ & M & Beni-Mellal & Male prisoners & M & 25 & $B$ & B \\
\hline S3LBB & $\mathrm{L}$ & Safi & NA & & & B & B \\
\hline $\mathrm{S} 2 \mathrm{J1BB}$ & $J 1$ & Marrakech & Male prisoners & $M$ & 28 & $B$ & $B$ \\
\hline S136BB & 36 & Agadir & Patients with STI's & $F$ & 35 & $B$ & B \\
\hline S1B1BB & $\mathrm{B} 1$ & Agadir & Female SW in MC & $\mathrm{F}$ & 28 & $B$ & $B$ \\
\hline $\mathrm{S1C1BB}$ & $\mathrm{C} 1$ & Agadir & Patients with STI's & F & 30 & $B$ & $B$ \\
\hline S1D1BB & D1 & Agadir & Marines & M & 28 & B & B \\
\hline S1E1BB & E1 & Agadir & Pregnant women & $\mathrm{F}$ & 38 & $B$ & $B$ \\
\hline S1F1BB & F1 & Agadir & Female SW in prison & $F$ & 21 & $B$ & $B$ \\
\hline S1G1BB & G1 & Agadir & Female SW in prison & $\mathrm{F}$ & 21 & $B$ & $B$ \\
\hline S1RBB & $\mathrm{R}$ & Agadir & Patient with $\mathrm{TB}$ & M & 20 & $B$ & $B$ \\
\hline S1VBB & V & Agadir & Male prisoners & M & 24 & $B$ & $B$ \\
\hline S1ZBB & Z & Agadir & Patients with STI's & $F$ & 18 & $B$ & $B$ \\
\hline S1V1BB & $\mathrm{V} 1$ & Taroudant & Pregnant women & $\mathrm{F}$ & 38 & $B$ & $B$ \\
\hline S235BB & 35 & Marrakech & Patients with STI's & M & 39 & $B$ & B \\
\hline $\mathrm{S} \times 2 \mathrm{~K} 1 \mathrm{O} 1 \mathrm{BB}$ & & Marrakech & NA & & & B & $B$ \\
\hline S211BB & 11 & Marrakech & Patients with STI's & $F$ & 23 & $B$ & $B$ \\
\hline $\mathrm{S} 2 \mathrm{~L} 1 \mathrm{BB}$ & $\mathrm{L} 1$ & Marrakech & Pregnant women & $\mathrm{F}$ & 27 & $B$ & $B$ \\
\hline $\mathrm{S} 2 \mathrm{M} 1 \mathrm{BB}$ & M1 & Marrakech & Male prisoners & M & 25 & $B$ & $B$ \\
\hline S2N1BB & N1 & Marrakech & Male prisoners & M & 22 & $B$ & B \\
\hline S1WBB & W & Agadir & Female SW in prison & $\mathrm{F}$ & 25 & $B$ & $B$ \\
\hline S115DB & 15 & Agadir & Male prisoners & M & 25 & $B$ & $B$ \\
\hline S1A1DB & $\mathrm{A} 1$ & Agadir & Male prisoners & M & 27 & $B$ & $B$ \\
\hline S114DB & 14 & Oulad Taima & Female SW in $\mathrm{MC}$ & $\mathrm{F}$ & 31 & $B$ & $B$ \\
\hline S118DB & 18 & Oulad Taima & Pregnant women & $F$ & 30 & $B$ & $B$ \\
\hline S3JBB & J & Safi & Male prisoners & M & 28 & $B$ & $B$ \\
\hline S29BB & 9 & Marrakech & Patients with STI's & $F$ & 22 & $B$ & $B$ \\
\hline S216BB & 16 & Marrakech & Patients with STI's & M & 40 & $B$ & $B$ \\
\hline $\mathrm{SX1 \textrm {K } 1 \mathrm { O } 1 \mathrm { BB }}$ & & Agadir & NA & 29 & 29 & $B$ & $B$ \\
\hline $\mathrm{S} 1 \mathrm{H} 1 \mathrm{BB}$ & $\mathrm{H} 1$ & Agadir & Pregnant women & $\mathrm{F}$ & 24 & $B$ & $B$ \\
\hline S225B & 25 & Marrakech & Male prisoners & $M$ & 29 & $B$ & \\
\hline S240B & 40 & Chichaoua & Patients with STI's & $\mathrm{F}$ & 34 & $B$ & \\
\hline S28B & 8 & Chichaoua & Patients with STI's & $\mathrm{F}$ & 44 & $B$ & \\
\hline S311B & 11 & Safi & Pregnant women & $\mathrm{F}$ & 26 & $B$ & \\
\hline S3KB & $\mathrm{K}$ & Safi & Patients with STI's & $\mathrm{F}$ & 41 & $B$ & \\
\hline S139B & 39 & Agadir & Patients with STI's & $\mathrm{F}$ & 25 & $B$ & \\
\hline S13B & 3 & Oulad Taima & Female SW in MC & $F$ & 29 & $B$ & \\
\hline S113 B & 13 & Oulad Taima & Female SW in MC & $\mathrm{F}$ & 39 & & $B$ \\
\hline N319CC & 19 & Meknes & Patients with STI's & $\mathrm{F}$ & 21 & $C$ & C \\
\hline
\end{tabular}


Table 1 Database of HIV sequences included in the study (Continued)

\begin{tabular}{|c|c|c|c|c|c|c|c|}
\hline N124AC & 24 & Tanger & Drug users & $M$ & 27 & A & $C$ \\
\hline N220BC & 20 & Rabat & Patient with TB & $\mathrm{F}$ & 33 & $B$ & $C$ \\
\hline N241AAE & 41 & Rabat & Patients with STI's & $\mathrm{F}$ & 25 & A & $\mathrm{AE}$ \\
\hline$\overline{\mathrm{N} 3 \mathrm{HAGAG}}$ & $\mathrm{H}$ & Meknes & Pregnant women & $\mathrm{F}$ & 22 & $\mathrm{AG}$ & $\mathrm{AG}$ \\
\hline N229AGAG & 29 & Rabat & Patients with STI's & $\mathrm{F}$ & 26 & $A G$ & $A G$ \\
\hline$\overline{\mathrm{N} 2 \mathrm{QAGAG}}$ & Q & Rabat & Patients with STI's & $\mathrm{F}$ & 29 & $\mathrm{AG}$ & $\mathrm{AG}$ \\
\hline $\mathrm{N} 2 \mathrm{OAG}$ & $\mathrm{O}$ & Rabat & Patients with STI's & $\mathrm{F}$ & 29 & $A G$ & \\
\hline N2PAG & $P$ & Rabat & Patients with STI's & $M$ & 28 & $\mathrm{AG}$ & \\
\hline N446AG & 46 & Oujda & Patients with STI's & $F$ & 40 & $A G$ & \\
\hline$\overline{\text { SITAGAG }}$ & $T$ & Agadir & Hotels workers & $M$ & 35 & $A G$ & $\mathrm{AG}$ \\
\hline S1SAGB & $\mathrm{S}$ & Agadir & Female SW in MC & $F$ & 30 & $A G$ & $B$ \\
\hline S27AGAG & 7 & Chichaoua & Consultant for STI & $\mathrm{F}$ & 24 & $\mathrm{AG}$ & $\mathrm{AG}$ \\
\hline S317AGAG & 17 & Safi & Consultant for STI & $\mathrm{F}$ & 39 & $\mathrm{AG}$ & $\mathrm{AG}$ \\
\hline
\end{tabular}

CFR02_AG. All Moroccan sequences with a confirmed subtype were assembled, and sequences from the same subject with concordant subtypes in PR and RT were concatenated. MLtrees were inferred using the final alignments for each subtype using concatenated PR/RT sequences. Analyses were performed assuming the GTR + Gamma model of nucleotide evolution. Statistical support was assessed by non-parametric bootstrapping (number of replicates $=500$ ) using PHYML.

\section{Molecular clock analysis}

The evolutionary rate (nucleotide substitutions per site per year) and the time of the most recent common ancestor ( $\mathrm{T}_{\mathrm{MRCA}}$, years) of HIV-1B in Morocco were inferred using sequences sampled at different time points by the MCMC approach implemented in BEAST [17]. The analyses were performed with the same nucleotide substitution model described in the previous section, and different coalescent priors (constant, exponential and Bayesian Skyline Plot), assuming a strict or a relaxed molecular clock [18]. An MCMC was run for 100,000,000 generations with sampling every $10,000^{\text {th }}$ generation. The results were visualized in Tracer. The effective sample size (ESS) value for each parameter was $>500$ indicating sufficient mixing of the Markov chain.

\section{Results}

Sixty HIV-1 positive sera were genotyped: 41 from the south (68.3\%), 14 (23.3\%) from the center and 5 (8.3\%) from the North of Morocco. Sex distribution was $62 \%$ females and $38 \%$ males, with an average age of 29 years. The risk groups represented in the study sample included persons attending STI clinics (38.6\%), male prisoners (17.5\%), female sex workers (15.8\%), pregnant women (14\%), people with TB (8.8\%), drug users (5.3\%), marines (1.7\%) and hotel workers (1.7\%) (Table 1).
The PR and RT sequences were both positive for 47 (78.3\%) samples. Of these, 43 (91.5\%) had concordant subtype assignments including $36(83.7 \%)$ subtype B, $6(14 \%)$ CRF02_AG and 1 (2.3\%) subtype C (Table 1 ). The remaining $4(8.5 \%)$ samples in which both PR and RT regions were positive revealed discordant subtypes that represent intersubtypes and/or inter-CRF recombinant viruses. They include B/C, A/C, A/CRF01_AE and CRF02_AG/B which are represented by one sample each. Finally, of the 13 specimens with HIV-1 subtype assignment for only one viral region, $8 \mathrm{PR}$ and $2 \mathrm{RT}$ sequences were of subtype $\mathrm{B}$ and 3 PR sequences were of subtype CRF02-AG.

HIV-1 subtypes appeared to be differently distributed in Moroccan geographic regions. Subtype B strains appeared to be widely distributed with little geographic compartmentalization from region to region, whereas the single samples of subtype $\mathrm{C}, \mathrm{A} / \mathrm{C}, \mathrm{B} / \mathrm{C}$ and $\mathrm{A} /$ CRF01_AE were all concentrated in the northern regions of Morocco.

Figure 1 shows a ML tree, including HIV-1 subtype B sequences from Morocco as well as worldwide reference sequences downloaded from the HIV databases (http:// www.hiv.lanl.gov/content/index). Overall, Moroccan strains are highly intermixed with reference strains from different geographic regions, suggesting multiple introductions of subtype B in Morocco over a relatively long period of time. Two highly supported monophyletic clades (100\% and $94.5 \%$ bootstrap support, respectively) of Moroccan strains appear to be related to HIV-1B sequences from Europe, whereas a third large clade clustered together with sequences from the United States, although the clade was only weakly supported by bootstrapping $(<50 \%)$. The time of the most recent common ancestor (TMRCA) of HIV-1B Moroccan strains calculated by molecular clock analysis dated back to 1983 (95\% high posterior density intervals: 1975 - 


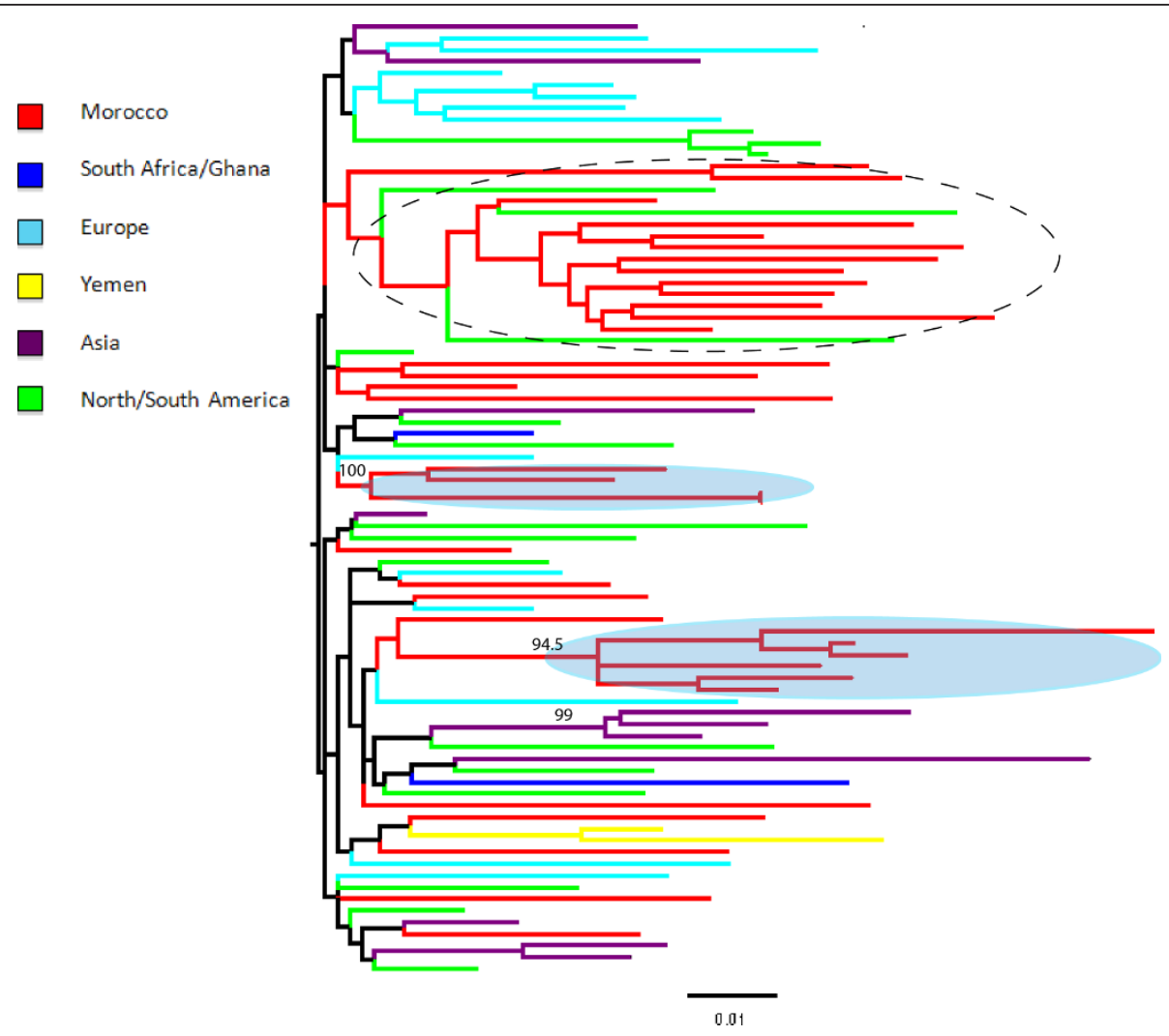

Figure 1 HIV-1B Maximum likelihood (ML) trees. The ML tree includes HIV-1B Moroccan sequences for which we have RT and PR sequences, as well as 46 subtype B reference sequences from the HIV database that were randomly chosen to represent major geographic areas in the world. The tree was generated using the GTR+G model of nucleotide substitution using the concatenated RT and PR genes. Branches are drawn in scale, according to the bar at the bottom, and colored to reflect geographic origin according to the legend of the figure. The number along a branch indicates significant bootstrap support (>65\%). Sequences were named using the year of sampling preceded by the two letter county code of origin, according to the HIV database guidelines.

1987) according to the constant population size coalescent prior enforcing a relaxed molecular clock. Different coalescence priors also produced very similar estimates (data not shown).

Figure 2 shows a ML tree of Moroccan and reference CRF02_AG strains available in HIV databases. In contrast to the subtype B ML tree, the Moroccan strains are highly localized in two distinct monophyletic clades related to sequences from Cameroon and Senegal. Although the result should be interpreted with caution, given the relatively small number of available sequences for phylogenetic comparison, the tree suggests two separate introductions of CRF02_AG in Morocco from sub-Saharan Africa, dated in 1995 and 1998 respectively, according to the constant population size coalescent prior enforcing a relaxed molecular clock. Again, different coalescence priors had little effect on the estimates (data not shown).

\section{Discussion}

We present the first data on the molecular epidemiology of HIV-1 in Morocco from the national HIV sentinel surveillance survey data. As of 2005, subtype B is still predominant (76.7\%), yet following subtype $B$, there is a high diversity of non-B subtypes, especially CRF02_AG recombinant (15\%). Geographic subtype repartition suggests the co-evolution of a more ancient diffusion of European subtype B, and of a more recent spread of sub-Saharan African strains in some Moroccan regions.

These results demonstrate a high diversity of HIV-1 strains in Morocco. This is different from what was reported in 1997 where the distribution of subtype B, A and $\mathrm{F}$ strains in Morocco were 93.5\%, 1.0\%, and 0.5\% respectively [12]. However, these results are consistent with our previous results [19] and the more recent results described from the Casablanca region [13]. These findings are also consistent with results described in other countries of the region, including the neighbouring West African countries [14]. The increase of HIV non-B subtypes was also recently reported in many Western Europe countries. Studies conducted in France, Spain, Switzerland, and Portugal have found that the proportion of non-B subtypes may exceed $20 \%[20,21]$. 


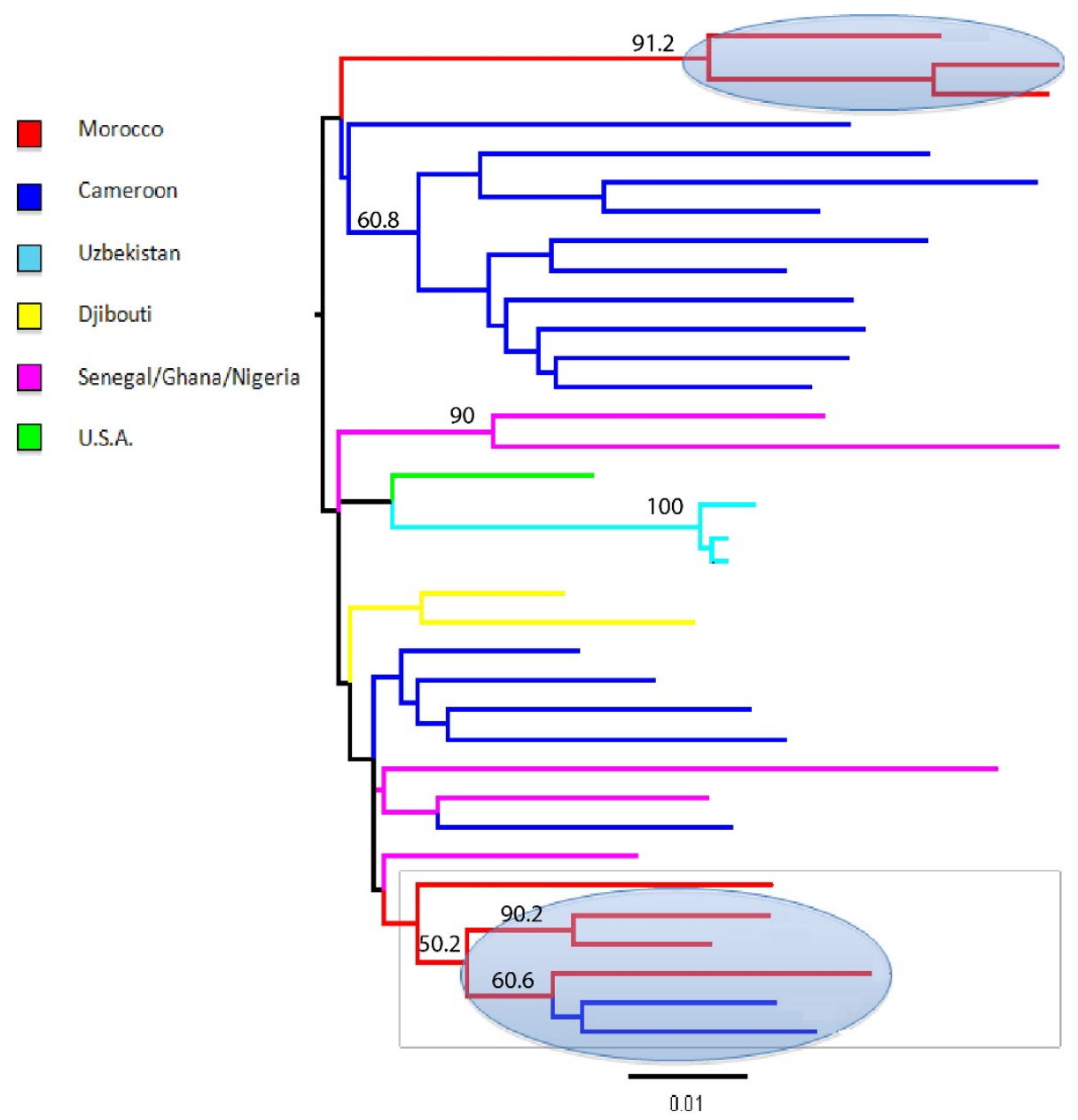

Figure 2 HIV-1 CRF02_AG Maximum likelihood (ML) trees. The ML tree includes HIV-1 Moroccan CRF02_AG sequences, for which we have RT and PR sequences, together with 28 CRF02_AG strains downloaded from the HIV databases for which the full genome sequences were available. The tree was generated using the GTR+G model of nucleotide substitution using the concatenated $R T$ and $P R$ genes. Branches are drawn in scale, according to the bar at the bottom, and colored to reflect geographic origin according to the legend of the figure. The number along a branch indicates significant bootstrap support ( $>65 \%$ ). Sequences were named using the year of sampling preceded by the two letter county code of origin, according to the HIV database guidelines.

CRF02_AG, which predominates in West Africa, is increasingly more prevalent among the non-B subtypes in these Western European countries.

The overall incidence of HIV-1 in Morocco has been increasing at approximately 15\% per year since 2000 . The increasing incidence of HIV combined with the identification of additional non-B subtypes raises concerns regarding the control of the current epidemic. The entry of new HIV recombinant viruses is likely the consequence of active exchange between different populations, such as Moroccan groups at risk and persons migrating through Morocco from sub-Saharan Africa. Before 1997, the presence of Sub-Saharan African individuals in Morocco was mostly limited to students and tourists. However, migration of people from subSaharan Africa to and through Morocco has been increasing since the late 1990s. In 2007, the Moroccan Ministry of the Interior estimated that approximately 15,000 irregular migrants flow through Morocco each year [22]. In response, the European Union has tightened its boarder control and immigration policies. As a result, many of the migrants settle in Morocco, waiting for an opportunity to cross into Europe. In addition, regular and irregular migrants face many economic and social issues that may increase their risk for HIV transmission. For example, issues such as human trafficking and prostitution could contribute to the circulation of non-B HIV subtypes such as CRF02_AG throughout the country.

The fact that subtype B was more distributed throughout the country, especially in the big-touristic cities (Agadir, Marrakech and Casablanca), suggest this 
subtype may reflect an older infection. Persons with subtype CRF02_AG were also widely distributed geographically; however, this subtype was not detected in Morocco before 1997, suggesting a more recent epidemic. These findings are also supported by our molecular clock analysis. The other non-B subtypes and recombinants represented more localised transmission due to $C$, $\mathrm{A} / \mathrm{C}, \mathrm{B} / \mathrm{C}$ and $\mathrm{A} / \mathrm{CRF} 01 \_\mathrm{AE}$ strains in the northern part of Morocco.

As the first case of HIV/AIDS in Morocco was reported in 1986, there is an excellent agreement with the TMRCA of HIV-1B of 1983 estimated by molecular clock analysis. Since that date, HIV has been spreading throughout the country, mainly by heterosexual transmission [11]. According to the sentinel surveillance system, the overall HIV prevalence is less than $1 \%$ in Morocco. However, even though Morocco is a low prevalence epidemic, HIV/AIDS cases are steadily rising, chiefly in the southern Morocco region of Agadir and neighbouring areas that may represent the epicentre of the epidemic within Morocco [23].

Our findings should be interpreted in light of study limitations. While our analysis included all HIV positive specimens from the 2004-2005 survey, the sample size was relatively small. Therefore, it is not possible to generalise the results as a national trend in Morocco. In addition, routes of transmission and clinical and immunologic status of the HIV-infected individuals were not available for this study, since they are not required in the surveillance process. However, the present data should prompt us to continue to track the molecular epidemiology of the HIV virus in Morocco at the national level. In this context, reinforcement of preventive measures to limit the spread of the epidemic is crucial. Lastly, by limiting our phylogenetic analysis to only the pol gene region, we may have missed some recombinants and therefore underestimated their distribution. However, our main finding that CRF02_AG is increasing in Morocco, signifying a shift from an epidemic previously dominated by serogroup B, remains true. In conclusion, the results of this study displayed that HIV diversity is more dynamic in Morocco and its pattern is shifting from the European to sub-Saharan one, i.e. with more subtypes non-B, namely the CRF02_AG. However, more studies to confirm the trend observed during this study and to better characterize the molecular HIV epidemic in Morocco will be of great importance. When taken together, these data demonstrate a dynamic evolution in the HIV diversity in Morocco. The emergence of new HIV subtypes are characterised by an important presence of non-B subtypes that appear to be linked to sub-Saharan populations. More data are needed to better understand the factors responsible for the introduction and spread of new HIV-1 subtype epidemics into regions where they did not exist previously.

\section{Abbreviations}

AIDS: Acquired Immune Deficiency Syndrome; CRF: circulating recombinant factor; ESS: effective sample size; HIV-1: Human Immunodeficiency Virus 1; IDU: injection drug users; MENA: Middle East and North Africa; ML: Maximum Likelihood; PCR: polymerase chain reaction; PR: Protease; RT: Reverse Transcriptase; STI: Sexually Transmitted Infections; TB: tuberculosis; TMRCA: Time to Most Recent Ancestor; WHO: World Health Organization

\section{Acknowledgements}

The authors acknowledge the staff of the National program to fight HIV/ AIDS, in the department of epidemiology and diseases control, Moroccan ministry of health; and all the staff working on HIV sentinel surveillance network, in Morocco. We thank also Dr Oumzil Hicham, Ms Imnae Belbacha, Dr Mengad Rajae, Ms Bennani Ouafae and Mr Amina Siwani from the National Reference Laboratory for HIV, National Institute of Hygiene. Finally, the authors thank all members of the molecular biology laboratory group who helped with this project. This study has a funding support from ESTHER Initiative, CNRST-INSERM Collaboration, and from the Emerging Pathogens Institute and the Center for AIDS Research at the University of Florida.

\section{Author details}

${ }^{1}$ Molecular Biology Unit, National Reference Laboratory for HIV, National Institute of Hygiene, Rabat, Morocco. ${ }^{2}$ HIV Diagnosis and Immunologic Follow up Unit, National Reference Laboratory for HIV, National Institute of Hygiene, Rabat, Morocco. ${ }^{3}$ Department of Zoology, University of Oxford, Oxford, UK. “Laboratoire de Virologie, Hôpital de la Croix Rousse, Lyon, France. ${ }^{5}$ Department of Epidemiology and Emerging Pathogens Institute, University of Florida, Gainesville, USA. ${ }^{6}$ Department of Pathology, Immunology and Laboratory Medicine, School of Medicine and Emerging Pathogens Institute, University of Florida, Gainesville, USA.

\section{Authors' contributions}

MA: Conduction of the study, samples collection, sequencing and analysis, preparation of the manuscript; SL: sample preparation, sequencing and analysis; EE: sample diagnosis and collection, HIV screening and confirmation, preparation of the manuscript; RG: phylogenetic analysis of samples and construction of phylogenetic trees; JCT: sequencing and analysis, preparation of the manuscript; RLC: interpretation of epidemiological data, preparation of the manuscript; MS: phylogenetic analysis of samples and construction of phylogenetic trees, preparation of the manuscript; PA: sequencing and analysis, preparation of the manuscript; TA: preparation of the manuscript; REA: conduction of the study, preparation of the manuscript. All authors have read and approved the final manuscript.

\section{Competing interests}

The authors declare that they have no competing interests.

Received: 22 September 2011 Accepted: 14 February 2012 Published: 14 February 2012

\section{References}

1. Leitner T, Korber B, Daniels M, Calef C, Foley B: HIV-1 subtype and circulating recombinant form (CRF) reference sequences. HIV sequence compendium 2005:41-48.

2. Los Alamos National Laboratory: HIV Sequence Database., Available at URL http://www.hiv.lanl.gov, accessed January, 2012.

3. Rambaut A, Posasa D, Crandall KA, Holmes EC: The causes and consequences of HIV evolution. Nature Reviews Genetics 2004, 5:52-61.

4. Salemi M: Toward a robust monitoring of HIV subtypes distribution worldwide. AIDS 2011, 25:713-714.

5. Robertson DL, Anderson JP, Bradac JA, Carr JK, Foley B, Funkhouser RK, Gao F, Hahn BH, Kalish ML, Kuiken C, Learn GH, Leitner T, McCutchan F, Osmanov S, Peeters M, Pieniazek D, Salminen M, Sharp PM, Wolinsky S, Korber B: HIV-1 nomenclature proposal. Science 2000, 288:55-56.

6. Robertson DL, Hahn BH, Sharp PM: Recombination in Aids viruses. J mol evolution 1995, 11:1423-1425. 
7. Simon $F$, Mauclère $P$, Roques $P$, Loussert-Ajaka I, Müller-Trutwin $M C$, Saragosti S, Georges-Courbot MC, Barré-Sinoussi F, Brun-Vézinet F: Identification of a new human immunodeficiency virus type 1 distinct from group M and O. Nat Med 1998, 4:1032-1037.

8. Thomson MM, Najera R: Travel and the introduction of human immunodeficiency virus type 1 non B subtypes genetic forms into Western countries. Clinical infectious Diseases 2001, 32:1732-1737.

9. Gray RR, Tatem AJ, Lamers S, Hou W, Laeyendecker O, Serwadda D, Sewankambo N, Gray RH, Wawer M, Quinn TC, Goodenow MM, Salemi M: Spatial phylodynamics of HIV-1 epidemic emergence in east Africa. AIDS 2009, 23(14):F9-F17.

10. Joint United Nations Programme on HIV/AIDS (UNAIDS): Report on the global AIDS epidemic, UNAIDS website. Available at: http://www.unaids.org/ globalreport/. Accessed January, 2012.

11. Elharti E, Alami M, Khattabi H, Bennani A, Zidouh A, Benjouad A, El Aouad R: Some characteristics of the HIV epidemic in Morocco. Eastern Mediterranean Health Journal 2002, 8:141-149.

12. Elharti E, Elaouad R, Amzazi S, Himmich H, Elhachimi Z, Apetrei $C$ Gluckman JC, Simon F, Benjouad A: HIV-1 diversity in Morocco. AIDS 1997, 11(14):1781-1783.

13. Bakhouch K, Oulad-Lahcen A, Bensghir R, Blaghen M, Elfilali KM, Ezzikouri S, Abidi O, Hassar M, Wakrim L: The prevalence of resistance-associated mutations to protease and reverse transcriptase inhibitors in treatmentnaïve (HIV)-infected individuals in Casablanca, Morocco. J Infect Dev Ctries 2009, 3(5):380-391.

14. Mumtaz G, Hilmi N, Akala FA, Semini I, Riedner G, Wilson D, Abu-Raddad L: HIV-1 molecular epidemiology evidence and transmission patterns in the Middle East and North Africa. Sex Transm Infect 2011, 87:101-106.

15. Descamps D, Delaugerre C, Masquelier B, Ruffault A, Marcelin AG, Izopet J, Chaix ML, Calvez V, Brun-Vézinet F, Costagliola D: Repeated HIV-1 resistance genotyping external quality assessments improve virology laboratory performance. J Med Virol 2006, 78(2):153-60.

16. Guindon S, Dufayard JF, Lefort V, Anisimova M, Hordijk W, Gascuel O: New algorithms and methods to estimate maximum-likelihood phylogenies: Assessing the performance of PhyML 3.0. Systematic Biology 2010, 59(3):307-21.

17. Drummond AJ, Rambaut A: BEAST: Bayesian evolutionary analysis by sampling trees. BMC Evol Biol 2007, 7:214

18. Drummond AJ, Ho SY, Phillips MJ, Rambaut A: Relaxed phylogenetics and dating with confidence. PLOS Biol 2006, 4(5):e88.

19. Akrim M, Adnaoui M, Himmich H, Khattabi H, Cotte L, André L, El Aouad R: Dynamic of distribution of HIV-1 subtypes in Morocco. Presented at: XVI International AIDS Conference Toronto; 2006, Available at URL http://www. iasociety.org/Abstracts/A2197144.aspx, accessed January, 2012.

20. Palma AC, Araújo F, Duque V, Borges F, Paixão MT, Camacho R, Portuguese SPREAD Network: Molecular epidemiology and prevalence of drug resistance-associated mutations in newly diagnosed HIV-1 patients in Portugal. Infection, Genetics and Evolution 2007, 7:391-398.

21. Perrin L, Kaiser L, Yerly S: Travel and spread of HIV-1 genetic variants. The lancet Infectious Diseases 2003, 3:22-27.

22. Consortium for Applied Research on International Migration (CARIM): CARIM - Migration Profile: Morocco 2009, Available at: http://www.carim.org/public/ migrationprofiles/MP_Morocco_EN.pdf, accessed January, 2012.

23. Elharti E, Zidouh A, Mengad R, Bennani O, El Aouad R: Monitoring HIV through sentinel surveillance in Morocco. Eastern Mediterranean Health Journal 2002, 8:819-25.

doi:10.1186/1742-6405-9-5

Cite this article as: Akrim et al:: HIV-1 Subtype distribution in morocco based on national sentinel surveillance data 2004-2005. AIDS Research and Therapy 2012 9:5.

\section{Submit your next manuscript to BioMed Central and take full advantage of:}

- Convenient online submission

- Thorough peer review

- No space constraints or color figure charges

- Immediate publication on acceptance

- Inclusion in PubMed, CAS, Scopus and Google Scholar

- Research which is freely available for redistribution

Submit your manuscript at www.biomedcentral.com/submit
Biomed Central 